Research Paper:

\title{
Effect of an Eight-week High-intensity Interval Training Program on Serum Cardiac Troponin I Level of Streptozotocin-induced Diabetic Rats
}

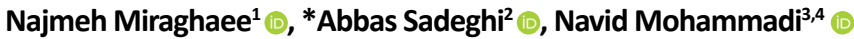

1. Department of Sport Sciences, Allameh Qazvini Institute of Higher Education, Qazvin, Iran.

2. Department of Physical Education and Sport Sciences, Faculty of Social Sciences, Imam Khomeini International University, Qazvin, Iran.

3. Children Growth Research Center, Research Institute for Prevention of Non-communicable Diseases, Qazvin University of Medical Sciences, Qazvin, Iran.

4. Canada Optimax Access Consultation, Ottawa, Canada.

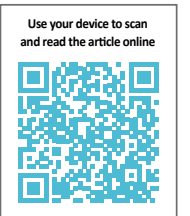

Citation Miraghaee N, Sadeghi A, Mohammadi N. Effect of an Eight-week High-intensity Interval Training Program on Serum Cardiac Troponin I Level of Streptozotocin-induced Diabetic Rats. Journal of Inflammatory Diseases. 2020; 24(5):386-397. https://doi. org/10.32598/JQUMS.24.5.1

doil ${ }^{\circ}$ https://doi.org/10.32598/JQUMS.24.5.1

Received: 23 Aug 2020

Accepted: 13 Oct 2020 Available Online: 01 Dec 2020

Keywords:

Heart, Diabetes, Cardiac troponin, High-intensity interval training

\begin{abstract}
Background One of the complications of diabetes, as a chronic metabolic disorder, is cardiovascular diseases. Objective This study aims to investigate the effect of an eight-week High-Intensity Interval Training (HIIT) program on serum Cardiac troponin I (Ctnl) level in streptozotocin-induced diabetic rats.

Methods In this experimental clinical trial, 30 rats were randomly assigned into three groups of healthy Control (C), Diabetic (D), and diabetic+training (D+T). The third group performed the training which included a treadmill running at an intensity of 85\%-90\% of maximum speed in 6-12 sessions of 2 min for 8 weeks, 5 days per week. Blood glucose level and high-sensitive Ctnl levels were measured 48 hours after the last training session and 12 hours of fasting. One-way ANOVA and Tukey's test were used to analyze the collected data, considering a significance level of $\mathrm{P}<0.05$.

Findings The induction of diabetes caused a significant increase in blood glucose $(P=0.01)$ and high-sensitive $\mathrm{Ctnl}(\mathrm{P}=0.01)$ levels in the $\mathrm{D}$ group. Also, a significant difference was observed in the blood glucose level of $D+T$ group compared to the $D$ group $(P=0.001)$. The $\mathrm{Ctnl}$ level also slightly reduced $(11 \%)$ in the $D+T$ group compared to the $D$ group, but it was not statistically significant $(P=0.591)$.

Conclusion he HIIT program can reduce the fasting blood glucose and increase the serum level of highsensitive Ctnl to some extent in diabetic rats; therefore, it can be an appropriate strategy for diabetics. However, there is a need for more studies in this area.
\end{abstract}

\section{Extended Abstract}

\section{Introduction}

$\mathrm{n}$ addition to having numerous complications, diabetes increases the risk of coronary heart disease and death [2]. Identifying people at risk of cardiovascular diseases based on specific criteria can improve their monitoring and treatment. One of the most important indicators is Cardiac
Troponin I (CtnI) as a biomarker of myocardial damage [5] . Cardiac troponins increase in myocardial damage earlier than other paraclinical markers and remain in the blood longer [7]. The American College of Sports Medicine (ACSM) has recommended exercise as a best way to prevent heart disease. Adaptation to aerobic exercise is associated with increased maximum oxygen consumption, cardiac output and ultimately left ventricular mass size [12]. Due to the relationship between physical activity and cardiorespiratory fitness, determining the effect of duration and intensity

* Corresponding Author:

Abbas Sadeghi

Address: Department of Physical Education and Sport Sciences, Faculty of Social Sciences, Imam Khomeini International University, Qazvin, Iran. Tel: +98 (28) 33901782

E-Mail: sadeghi@soc.ikiu.ac.ir 
of exercise on the risk index of myocardial infarction can help provide an appropriate model for improving the health of people and prevent cardiovascular diseases, especially in diabetic patients.

\section{Materials and Methods}

This experimental study was conducted for this study according to the ethical guidelines of the Declaration of Helsinki on 30 male Wistar rats (Weight $=225-300$ $\mathrm{g}$; age $=3$ months) randomly divided into three groups of 10 rats including healthy Control (C), Diabetic (D), and Diabetic+Training (D+T).

\section{Diabetes induction}

A high-fat diet was provided to the animals for two weeks. Then, they received a singles dose of $35 \mathrm{mg} / \mathrm{kg}$ Streptozotocin (STZ) by intraperitoneal injection after six hours of fasting [13]. A week after diabetes induction, blood samples were collected from their caudal vein. Those with a blood glucose level above $250 \mathrm{mg} / \mathrm{dL}$ were considered diabetic.

\section{Training protocol}

An incremental High-intensity Interval Training (HIIT) protocol on the treadmill was implemented to assess the maximum speed, starting at $10 \mathrm{~m} / \mathrm{min}$ and then increased by $3 \mathrm{~m} / \mathrm{min}$ every 2 minutes until exhaustion. The main exercise included 6-12 sessions of 2-min high-intensity exercise with a speed of $85-90 \%$ of maximum speed with 1-min low-intensity exercise at $10 \mathrm{~m} / \mathrm{min}$. The running speed increased by about $10 \%$ every week throughout the study. Since handling animals on the treadmill may induce stress, sedentary control animals were also trained on the treadmill once a week to familiarize with handling and treadmill environment [15].

\section{Blood glucose and high-sensitive CtnI assay}

All animals were intraperitoneally anesthetized with ketamine $(90 \mathrm{mg} / \mathrm{kg}-1)$ and xylazine $(10 \mathrm{mg} / \mathrm{kg}-1)$ and sacrificed $48 \mathrm{~h}$ after the last training session. A sufficient amount of blood was taken from the left ventricle of the heart by a syringe. All blood samples were immediately centrifuged (3000rpm, $15 \mathrm{~min}, 4^{\circ} \mathrm{C}$ ) and stored at $-80^{\circ} \mathrm{C}$ until analysis to extract the serum. Fasting blood sugar levels were measured by a colorimetric glucose-oxidase procedure. Serum CtnI level was measured by the ELISA method using a HighSensitive (HS) enzyme-linked assay kit specific for rat CtnI (Cloud-Clone Corp, USA), according to the instructions. All statistical analyses were performed in SPSS V. 24 software, considering a statistical significance level of $\mathrm{P}<0.05$.

\section{Results}

The Shapiro-Wilk test results showed that the data were normally distributed. The STZ-induced diabetes resulted in a significant increase in blood glucose $(\mathrm{P}=0.001)$ and HS-CtnI levels $(\mathrm{P}=0.001)$ in group $\mathrm{D}$. There was also a significant difference in blood glucose level in the $\mathrm{D}+\mathrm{T}$ group compared to group $\mathrm{D}(\mathrm{P}=0.001)$, indicating a significant decrease in fasting blood glucose level after exercise in this group. Although the HS-CtnI level in the D+T group slightly decreased $(11 \%)$ compared to the $\mathrm{D}$ group, this decrease was not significant $(\mathrm{P}=0.591)$.

\section{Discussion and Conclusion}

The results of this study showed that the 8 weeks of HIIT significantly reduced the blood glucose level of diabetic rats by $36.93 \%$. Recent studies that have used the HIIT in diabetic patients suggest that the exercise intensity may play a key role in managing diabetes [19]. The mechanisms for the effect of HIIT on the glycemic index of diabetic patients are not fully understood. The hepatic glucose production induced by exercise can also be improved following the fasting blood glucose reduction. On the other hand, the HIIT can use muscle fibers and deplete muscle glycogen faster [20]. Therefore, it may further increase the insulin sensitivity of muscle cells after exercise. It seems that the effectiveness of HIIT in increasing the insulin sensitivity and reducing the blood glucose level is higher.

In the present study, the induction of diabetes in rats increased the serum level of CtnI significantly. This finding suggests that diabetes can damage to the myocardial tissues $[22,34]$ The HIIT reduced the serum levels of $\mathrm{CtnI}$ in diabetic rats to some extent (11\%), which indicates the protective effect of this training program against cardiac tissue damage, although it was not significant. However, it is too early to comment definitively on the effect of such exercises on the specific markers of heart cell damage, and more research is needed to determine the impact of this type of exercise. Regular exercise increases myocardial adaptation and tolerance against ischemic injury [29]. The long-term exercise increases the activity of antioxidant enzymes in protecting the heart [31]. Other possible mechanisms are increased number of mitochondria, increased nitric oxide, and consequently increased endothelial nitric oxide synthase [33]. 


\section{Ethical Considerations}

Compliance with ethical guidelines

The research was approved by the Ethics Committee of the Allameh Qazvini Institute of Higher Education (Code: 97/K.A/1012) and the principles and codes of ethics in the research have been followed.

\section{Funding}

The present paper was extracted from the MA. thesis of the first author, Department of Sport Sciences, Allameh Qazvini Institute of Higher Education, Qazvin.

Authors' contributions

Conceptualization and finalization: Najmeh Miraghaee, Abbas Sadeghi and Navid Mohammadi; Methodology: Abbas Sadeghi; Data collection: Najmeh Mir Aghaee; Data analysis and interpretation: Abbas Sadeghi; Preparation of draft article: Najmeh Miraghaee; Critical editing and review of the text and content of the draft: Abbas Sadeghi and Navid Mohammadi; Project supervision and management: Abbas Sadeghi and Navid Mohammadi.

\section{Conflict of interest}

The authors declare no conflict of interest.

\section{Acknowledgments}

The author would like thank the officials of Allameh Qazvini Institute of Higher Education and the Sarai Cell Research Clinic. 


\title{
تأثير هشت هفته تمرين تناوبى شديد (HS-CtnI) I IIIT) بر سطوح سرمى ترويونين قلبى موش هاى ديابتى القاشده با استريت تمريت تئوزوتوسين
}

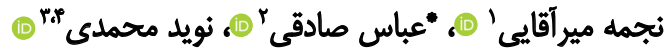 \\ 1. كروه علوم ورزشي، موسسه آموزش عالى علامه قزوينى، قزوين، ايران.

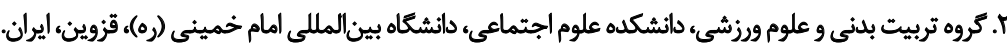

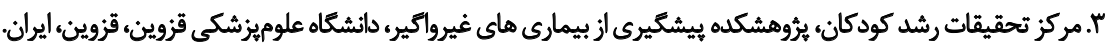

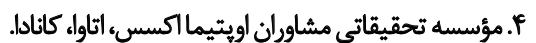

\begin{abstract}
إنمينه ديابت به عنوان يك اختلال متابوليكى منجر به بيمارى هاي قلبى عروقى مى شود.

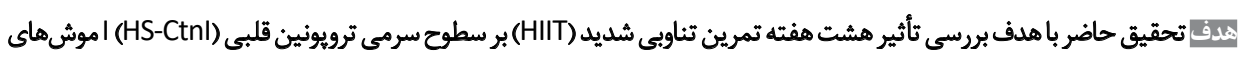

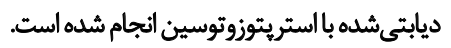

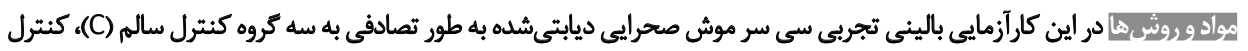

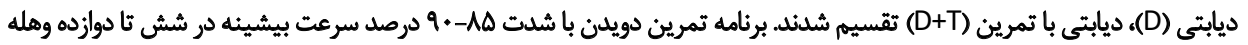

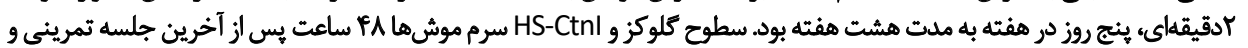

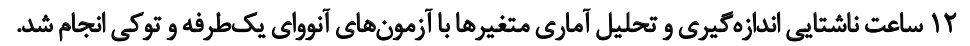

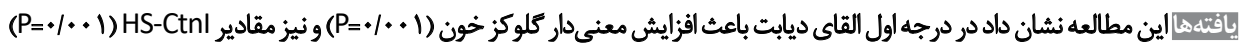

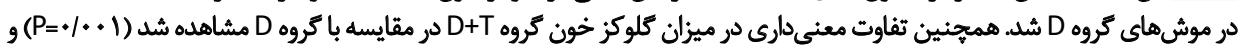

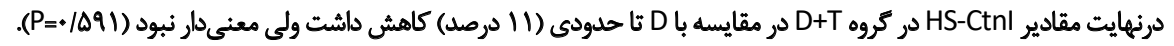

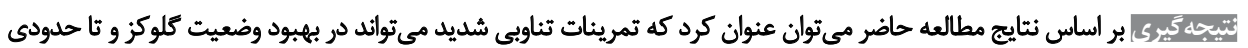

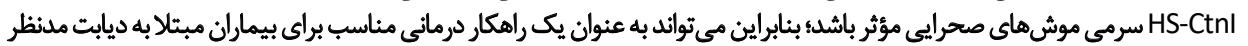

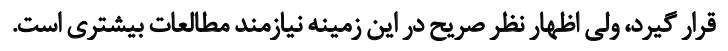

تاريخ دريافت: Y+شهريور 99

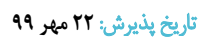

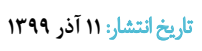

كاهش مركومير قلبي عروقي در بيمار ان مبتلابه ديابت باتوجه

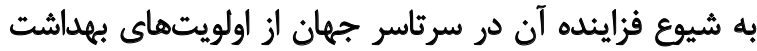

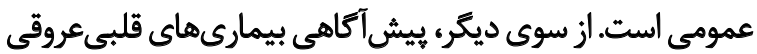

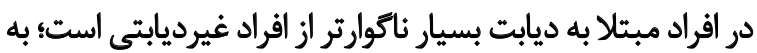

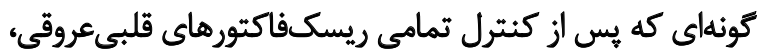

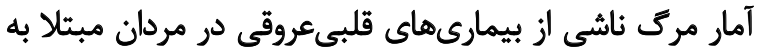

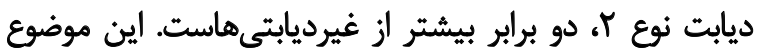

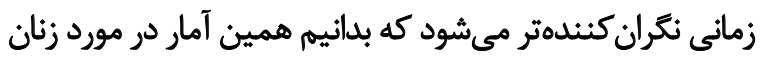

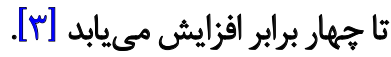
كزارشها نشان داده كه افزايش سيستماتيك قند خون در
مقال سرعت رو به رشد ديابت در جهان موجب شده است ثا اين

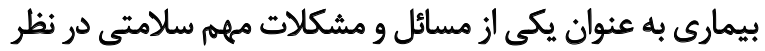

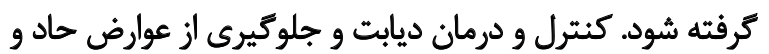

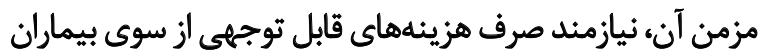

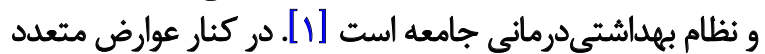

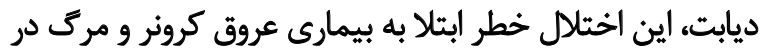

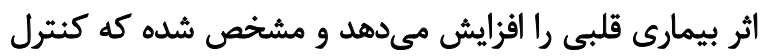

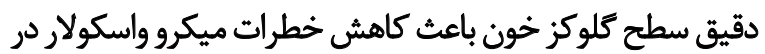
بيماران ديابتي ميىشود [ب].

$$
\begin{aligned}
& \text { * نويسينده مسيئول: }
\end{aligned}
$$

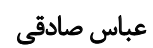

$$
\begin{aligned}
& \text { نشاني: قزويني، دانشكاه بين المللي امام حميني (ره)، دائشكده علوم اجتماعي، كروه تربيت بلدئي و علوم ورزشي. }
\end{aligned}
$$

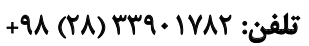

$$
\begin{aligned}
& \text { راياثامه: sadeghi@soc.ikiu.ac.ir }
\end{aligned}
$$




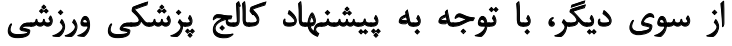

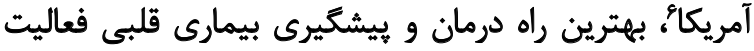

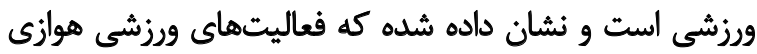

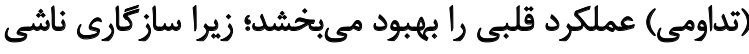

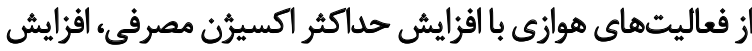

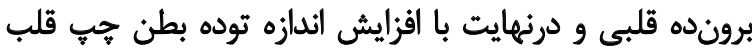

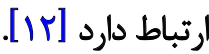

با توجه به ارتباط بين فعاليت بدنى و آمادتى قلبىتنفسى، بئي

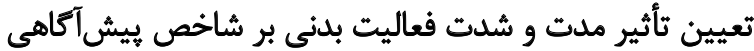

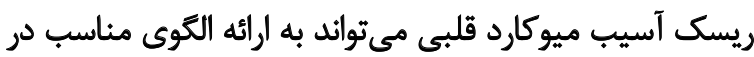

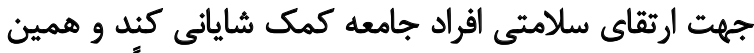

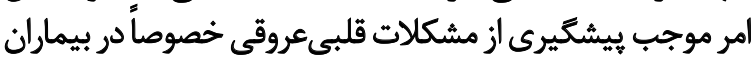

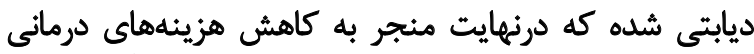

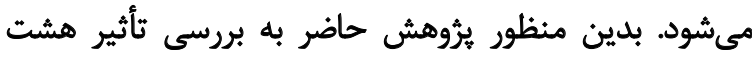

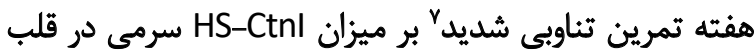

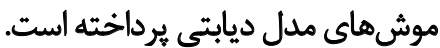

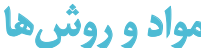

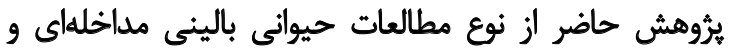

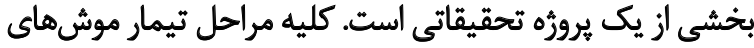

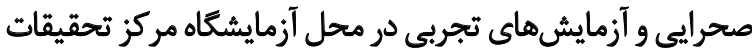

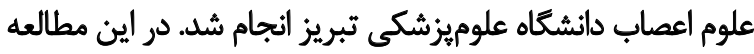

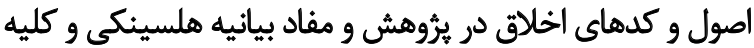

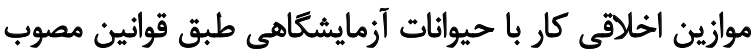

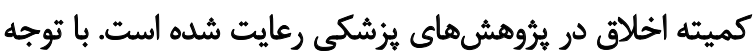

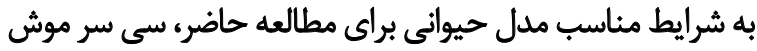

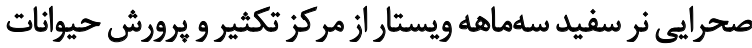

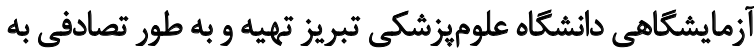

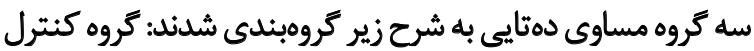
سالم (C)؛ كروه كنترل ديابتى (D) و كروه ديابتى +تمرين (D+T)

به منظور ايجاد حالت سازش با محيط، جلوكيرى از استرس

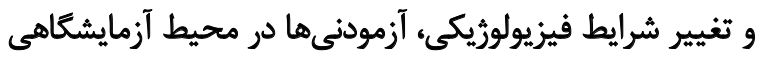

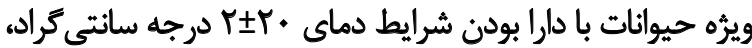

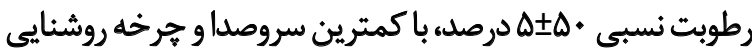

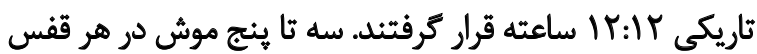

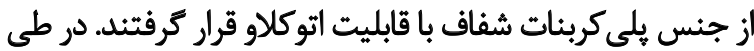

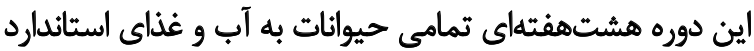

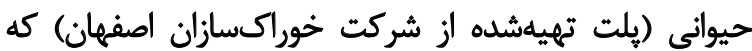
بهصورت دقيق اندازهكيرى و ثبت شده بوده، دسترسى آزاد داشتند.

6. American College of Sports Medicine (ACSM)

7. High-Intensity Interval Training (HIIT)
ديابت مليتوس مىتواند ضايعات شديدى را در ركهاى كوجك

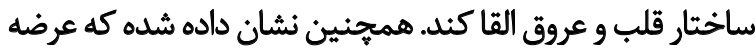

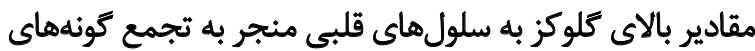

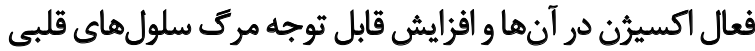

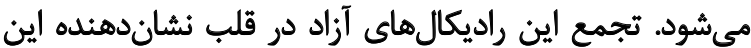

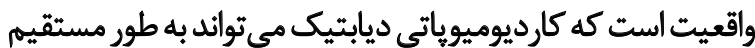

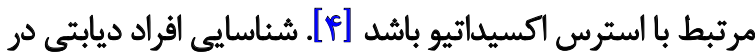

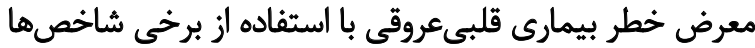

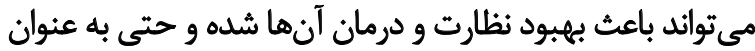

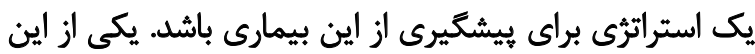

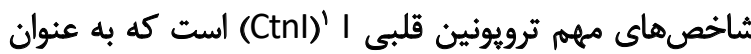

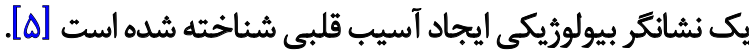

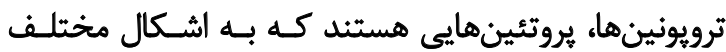

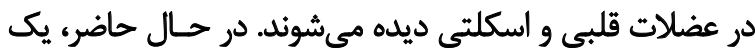

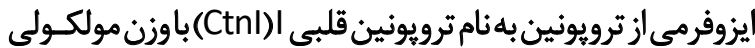

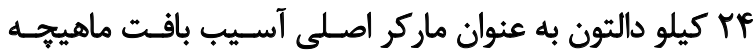

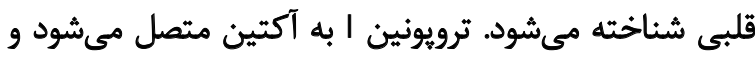

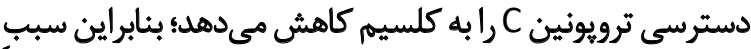

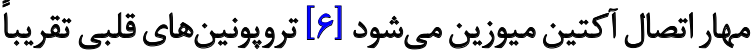

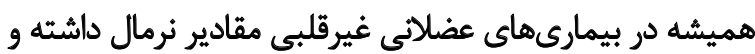

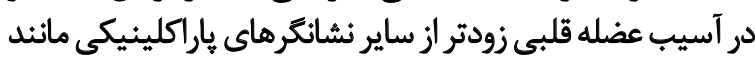

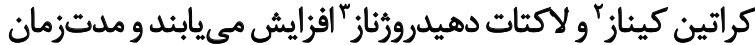
بيشترى در خون باقى مي مائند [V]

سنجش ترويونين قلبى اباحساسيت بالا' براى اندازمكيرى Ctnl

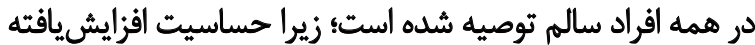

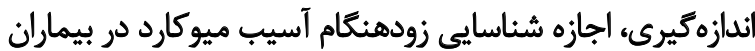

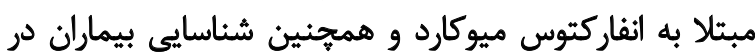

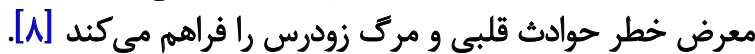

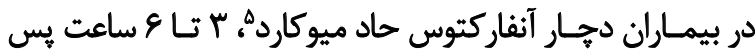

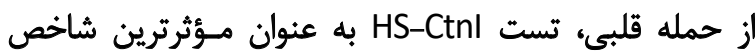

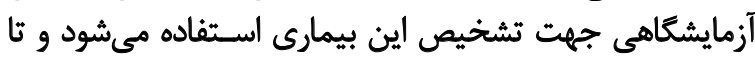

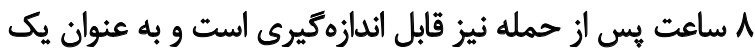

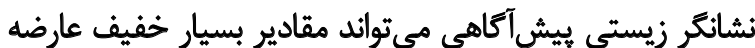

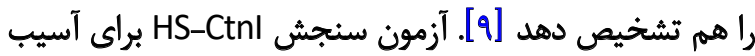

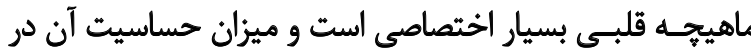

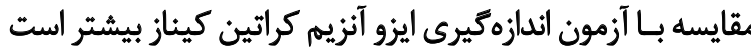

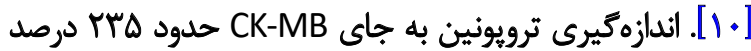

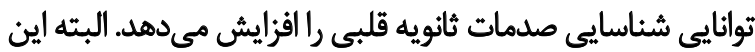

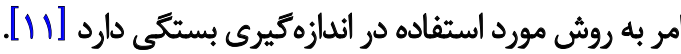

1. Cardiac Troponin I

2. Creatine Kinase Myocardial Band (CK-MB)

3. Lactate Dehydrogenase (LDH)

4. High-Sensitivity Cardiac Troponin I (HS-Ctnl)

5. Acute Myocardial Infarction (AMI) 
استراحت فعال كه شامل دويدنهاى ادامهدار روى نوار كردان با متان

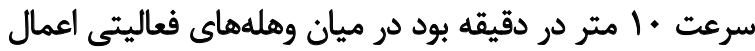

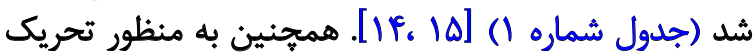

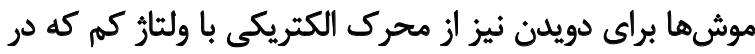

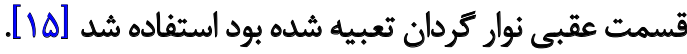

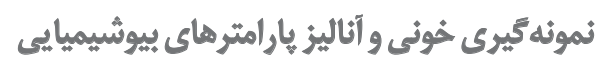

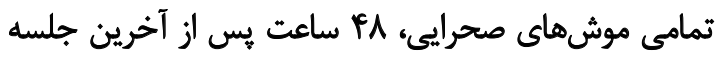

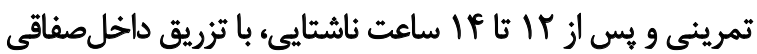
كتامين (•9 ميلى

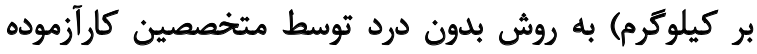

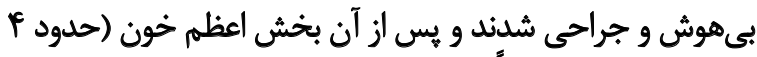

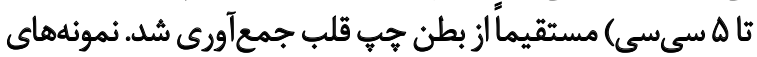

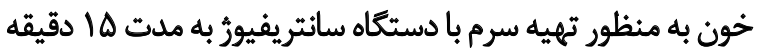

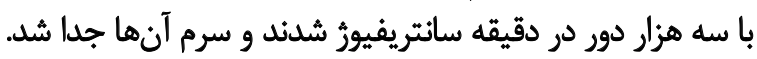

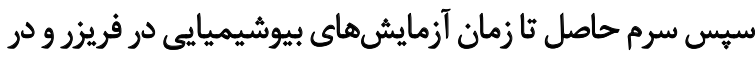

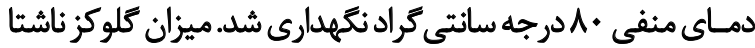

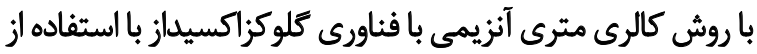

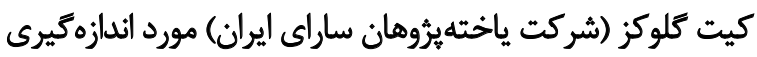

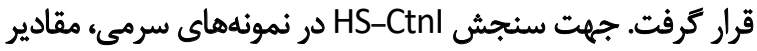

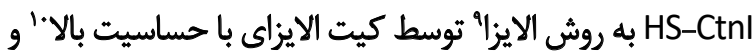

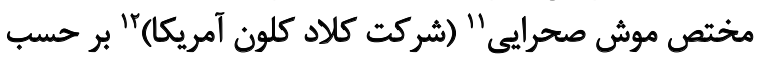

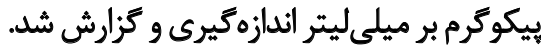

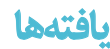

بر اساس نتايج حاصل از آزمسون شاييرو ويلك، اختلاف معنى دارى

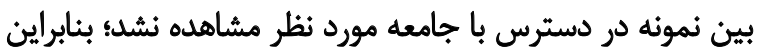

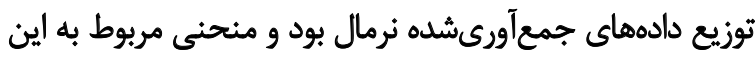

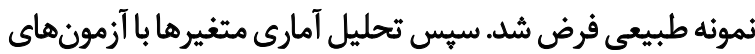

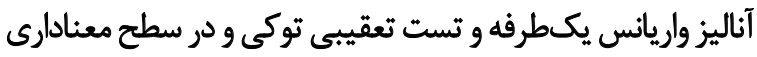

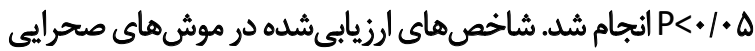
مورد مطالعه در جدول شماره باري ارائه شده است

نتايج حاصل از تحليل واريانس نشان داد كه القاى ديابت

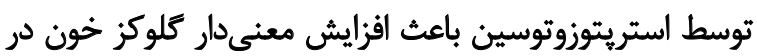

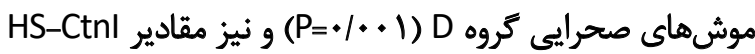

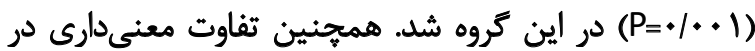

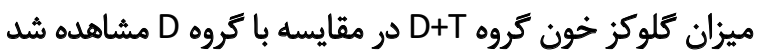

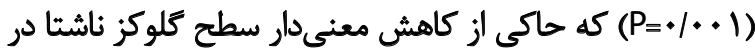

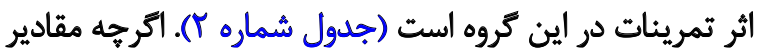
1I) در كروه DS-Ctnl

\section{ELISA}

10. High sensitive enzyme-liked assay kit.

11. Rat Cardiac Troponin I

12. Cloud-Clone Corp, USA

\section{روش ديابتى كردن موشها}

يس از كذشت دو هفته از شرايط سازكارى موشها با محيط

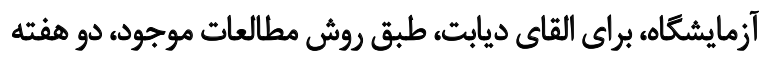

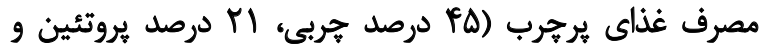

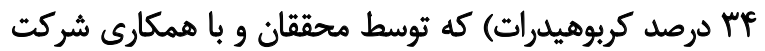

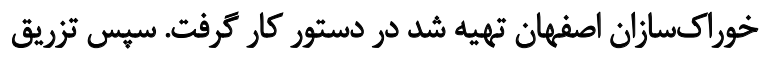

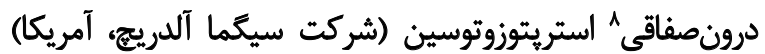

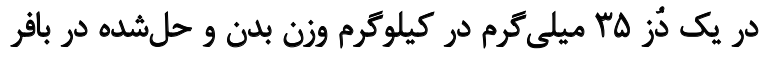

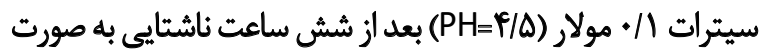

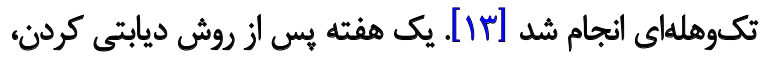

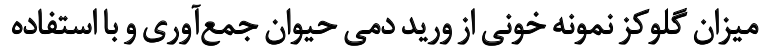

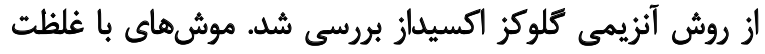

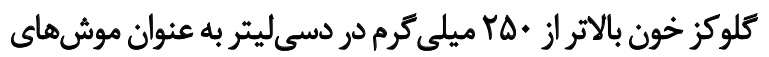

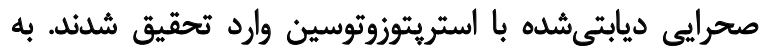

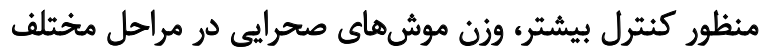

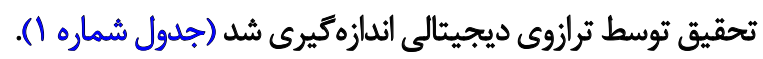

\section{يوروكل تمريني (HIIT)}

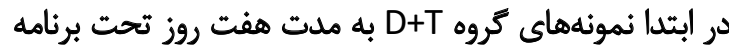

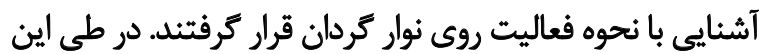

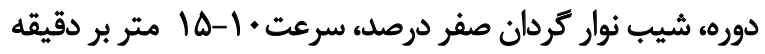

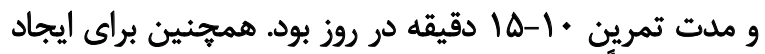

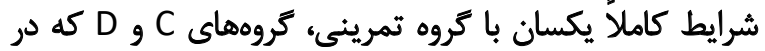

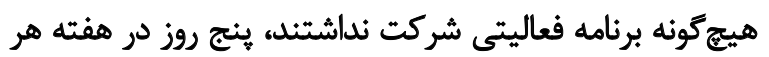

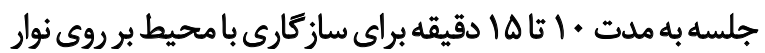

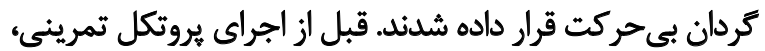

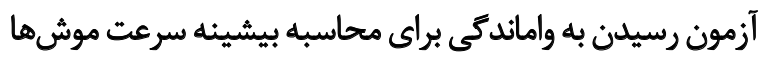

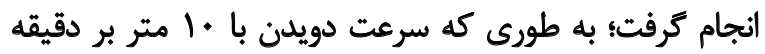

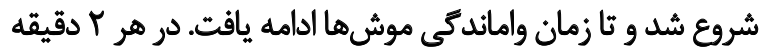

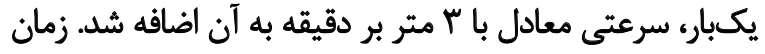

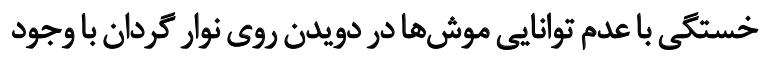

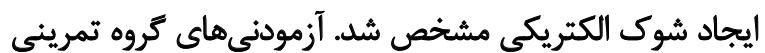

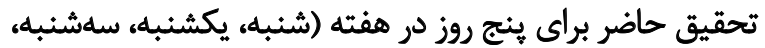

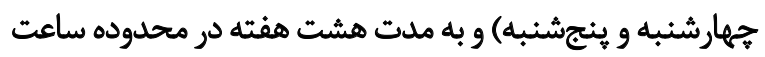

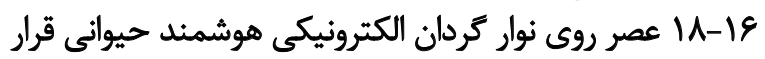

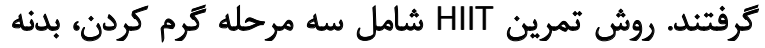

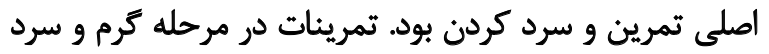

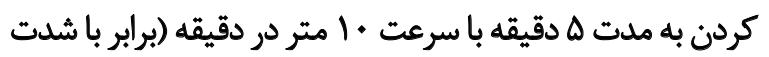

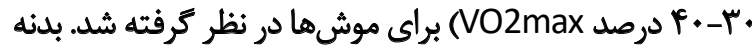

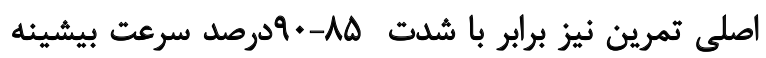

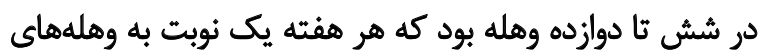

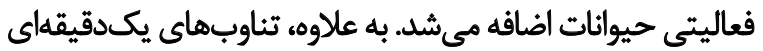

8. Intra Protaneal (IP) 
جدول (. وزن اوليه (قبل از مداخله) و وزن ثائويه (بعداز مداخله و قبل قربانى كردن) در بين كروههاي مورد مطالعه ميانكين

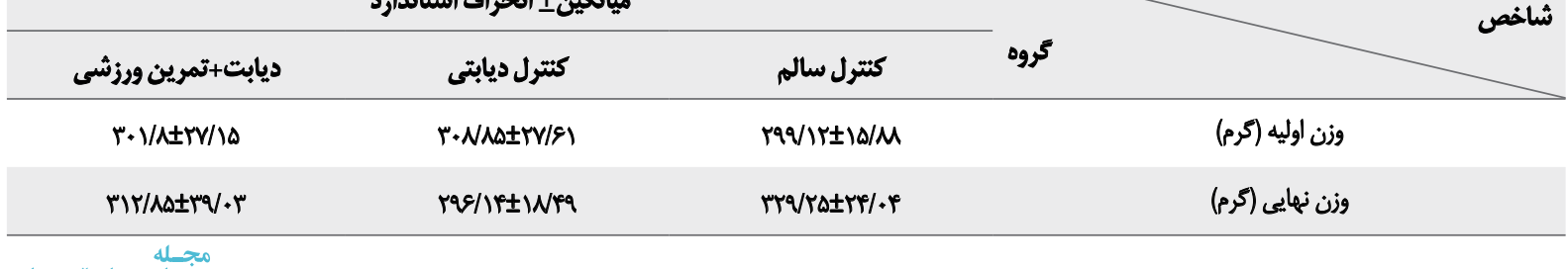

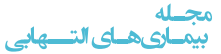

جدول ז.نتايج آزمون تحليل واريانس يكسرفه HS-CtnI و كلوكز خون

\begin{tabular}{|c|c|c|c|c|c|}
\hline سطح معنى دارى & $\mathbf{F}$ & درجه أزادى & ميانكين مجذورات & مجموع مجذورات & متغير \\
\hline$<+1 .+1$ & $q 1 / 1$. & r & $.97 / 18$ & MEFT & HS-Ctnl \\
\hline$<+1 .+1$ & fa/fe & r & ME/ITM & ar/aIAf & كلوكز خون \\
\hline
\end{tabular}

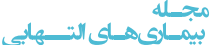

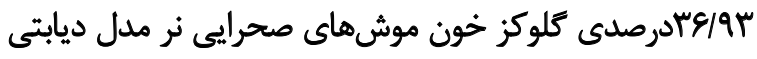

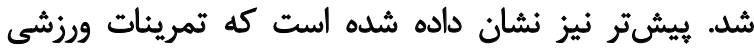

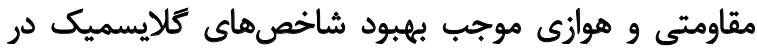

بيماران ديابتى ميىشود [IV]

اكرجه فعاليتهاى تناوبي با شدت بالا هماند فعاليتهاي

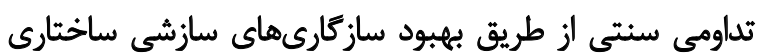

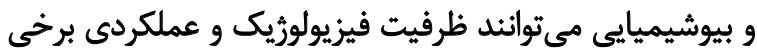

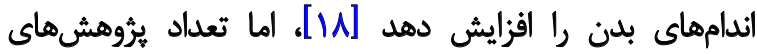

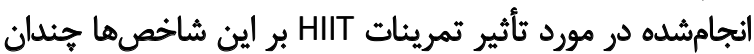

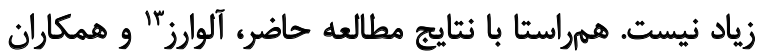

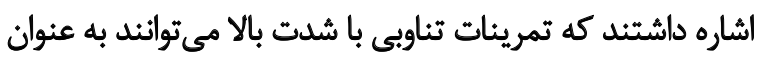
يك مداخله درمانى مفيد در بيماران ديابتى مورد استفاده قرار

\section{Alvarez}

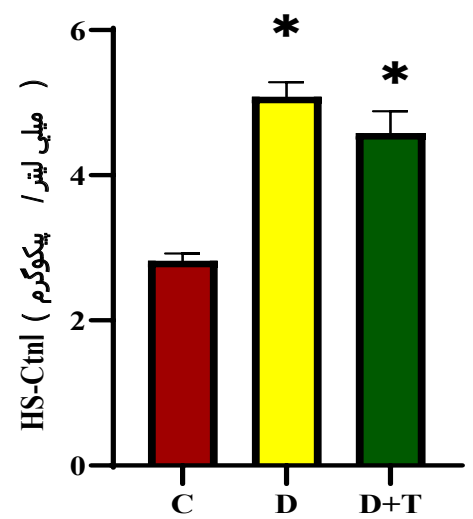

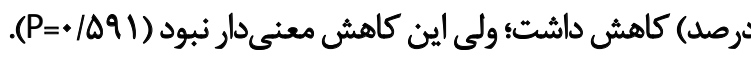

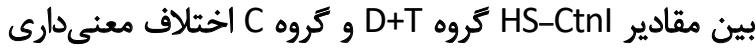

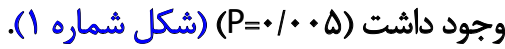

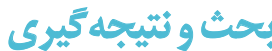

عوارض قلبىعروقى در بيماران مبتلا به ديابت شامل بيمـارى

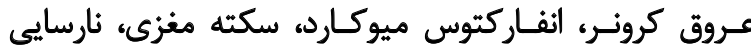

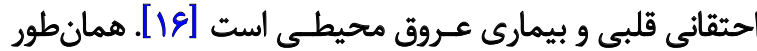

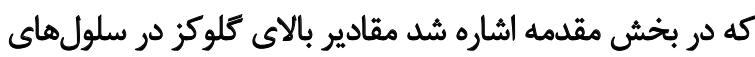

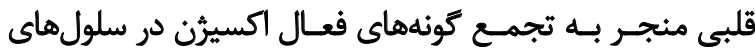

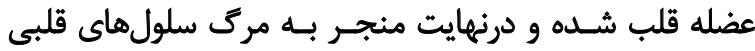

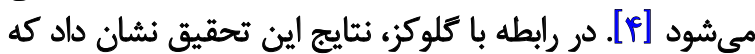

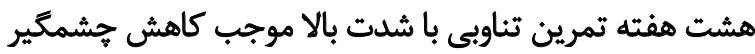

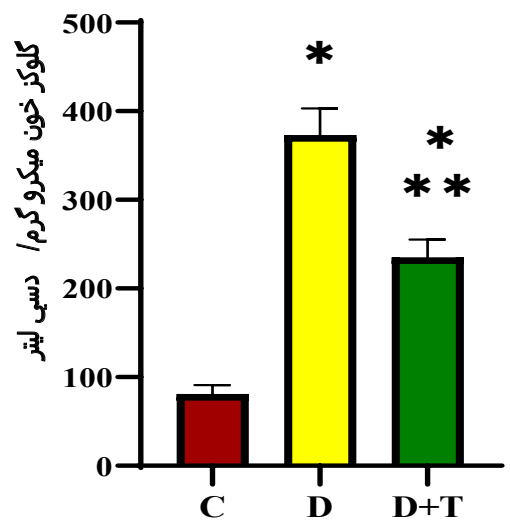




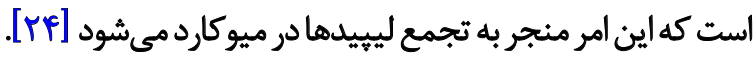

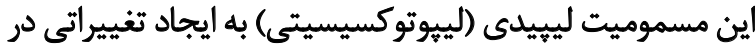

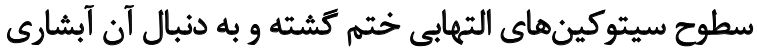

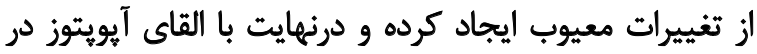

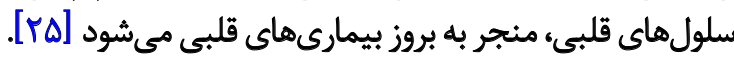
اعمال مداخله يروتكل تمرينى HIIT در كروه موشهاي

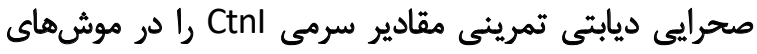

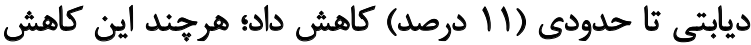

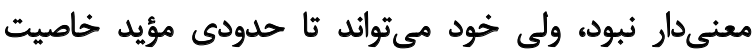

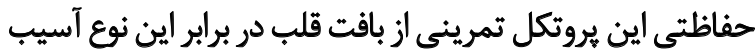

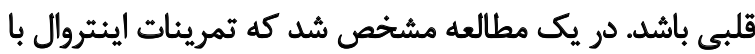

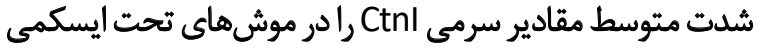

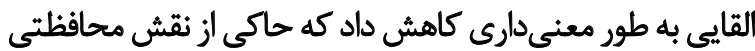

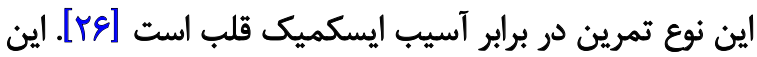

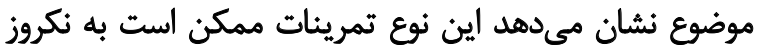

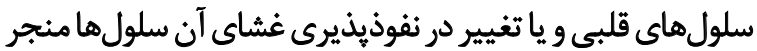

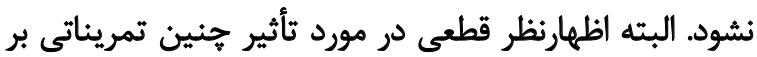

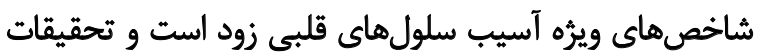
بيشترى لازم است تا آثار اين نوع تمرينات معلوم شود.

با وجود اين، افزايش سطوح سرمى Ctnl در ورزشكاران

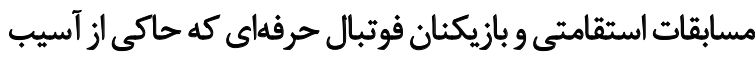

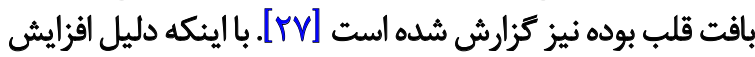

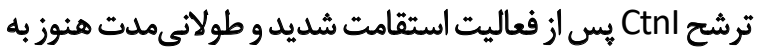

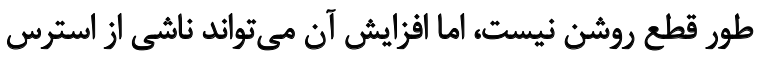

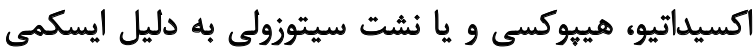

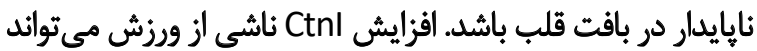

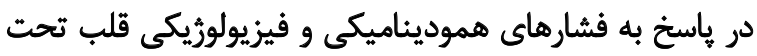

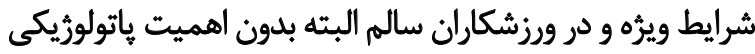

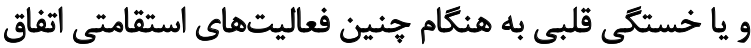

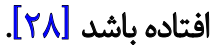

در حال حاضر مكانيسمهاى مسئول حفاظت قلبى تمرينات

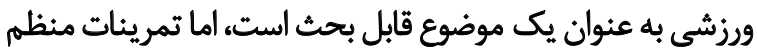

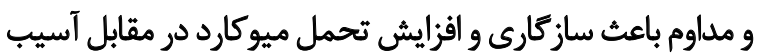

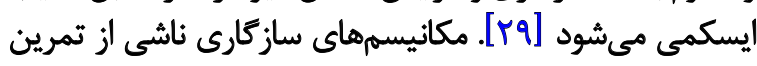

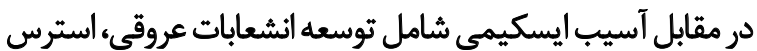

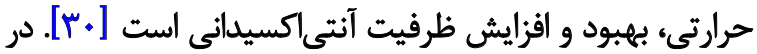

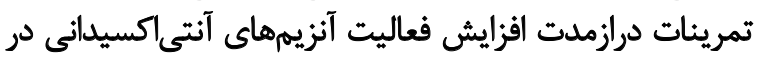

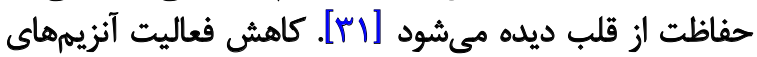

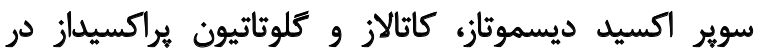
موشهاى تحت آسيب ايسكمى به طور معنى دارى توسط إنى انجام

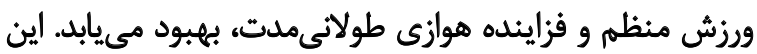

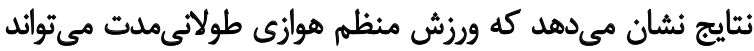

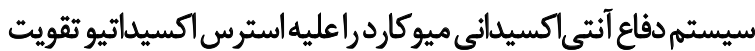

كيرند. نتايج مطالعه آنها حاكى ازٔ آن بود كه شانزده هفته تمرين

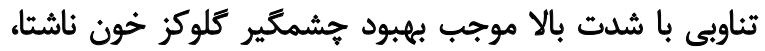

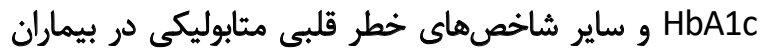

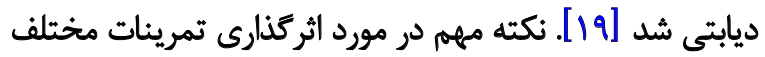

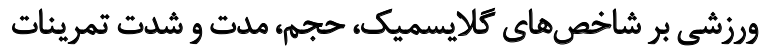

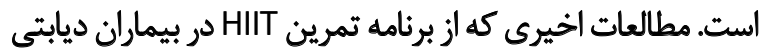

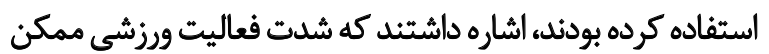

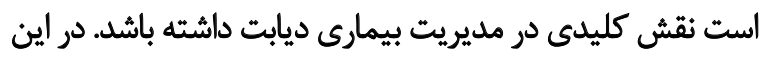

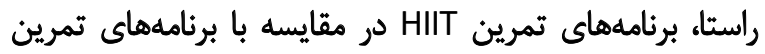

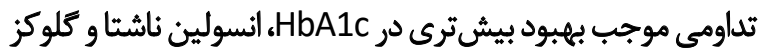

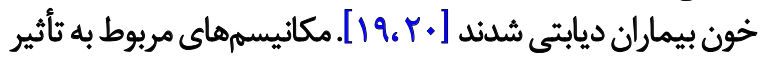

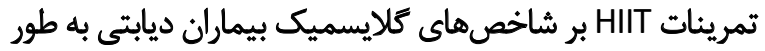

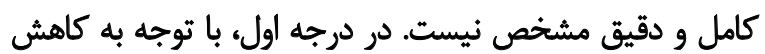

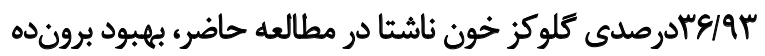

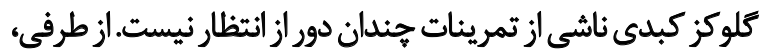

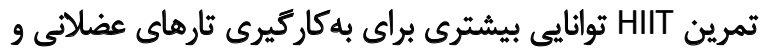

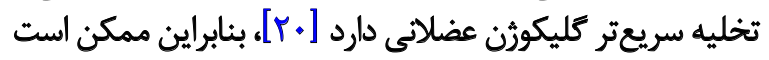

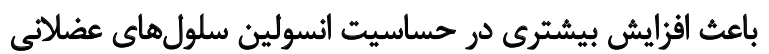

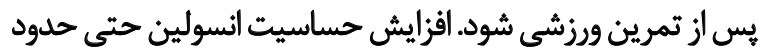

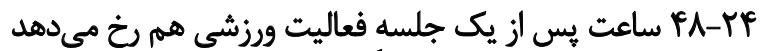

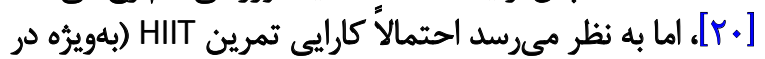

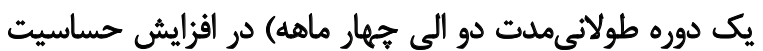
انسولين و كاهش كلوكز خون در بيماران ديابتى بالاتر باشد.

در مطالعه حاضر القاى ديابت در كروه موشهاي صدائ صحرايى

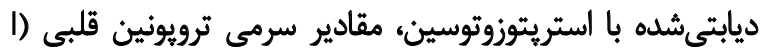

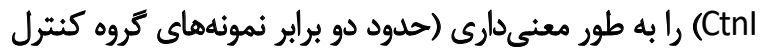

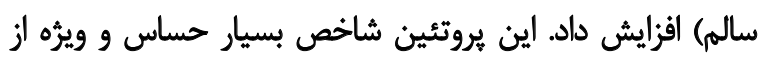

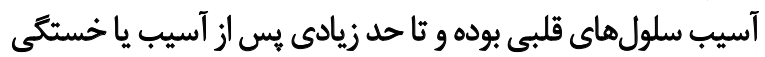

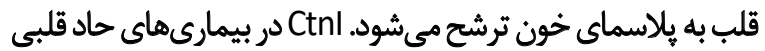

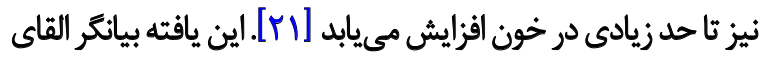

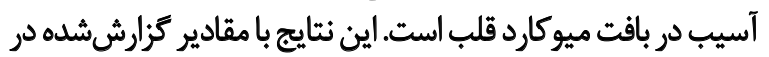

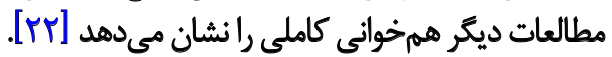

در خصوص ياتوفيزيولوري آسيبهاي قلبى ناشى از ديابت

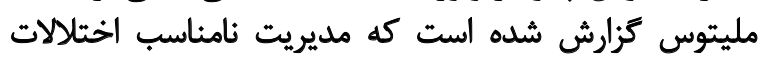

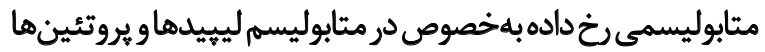

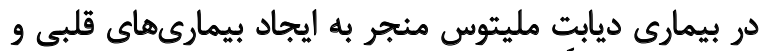

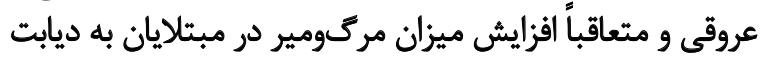

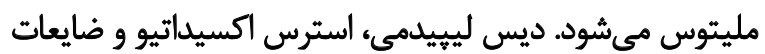

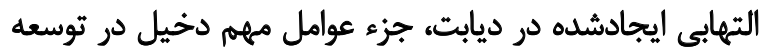

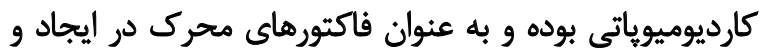

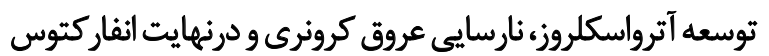

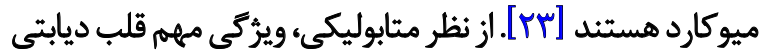
كاهش مصرف كلوكز و افزايش اكسيداسيون اسيدهاي هربي 
نجمه ميراقايى مصوب مؤسسه آموزش عالى علامه قزوينى بودهه و براى انجام آن از منابع مالى خاصى استفاده نشده است.

$$
\text { مشاركت ثويسندكًان }
$$

مفهومسازى مطالعه و نهاييىازى: نجمه ميرآقايى، عباس

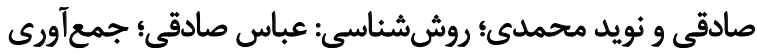

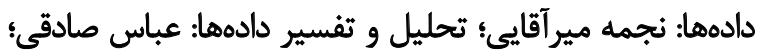

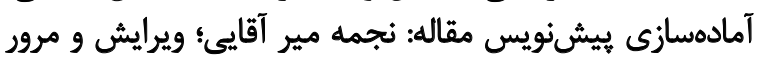

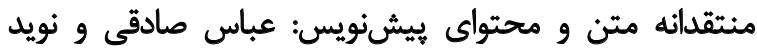
محمدى؛ نظارت و مديريت يرورث: عباس صادقى و نويد محمدى.

$$
\text { تعارض مثافع }
$$

بنابر اظهار نويسندكان اين مطالعه هيجكونه تعارض منافعى

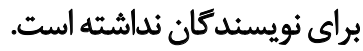

$$
\text { تشيكر و قدر دافي }
$$

بدينوسيله از مساعدت و همكارى صميمانه مسئولين

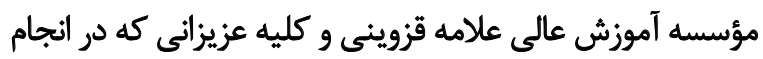

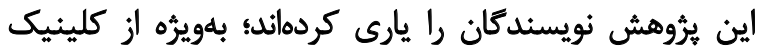

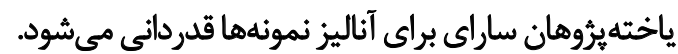

كند. همجنين، نشان داده شدهاست كه ميزان براكسيداسيون ليجيدى

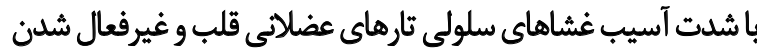

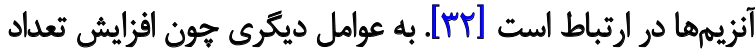

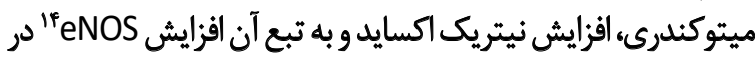

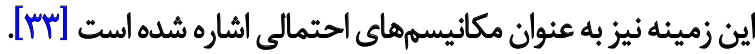
طبق بررسى هاى ماء اين تنها مطالعهاى است كه تاكنون در مورد اثر

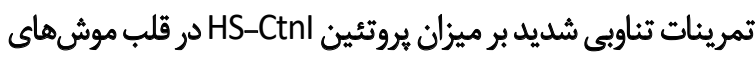

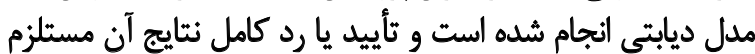

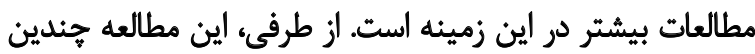

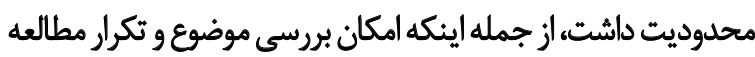

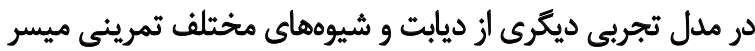

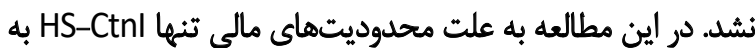

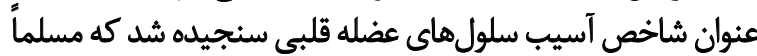

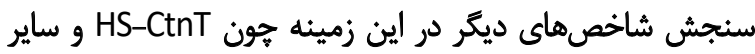

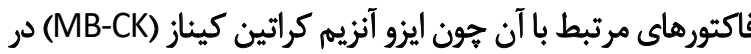
ارزيابى دقيقتر تشخيص، ايسكمى وريسك آنفاركتوس مؤثرتر است.

ابتلا به ديابت مليتوس با استريتوزوتوسين با القاى استرس

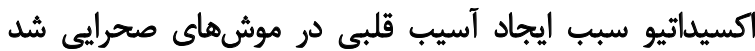

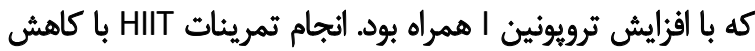

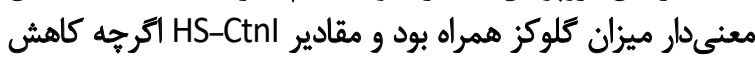

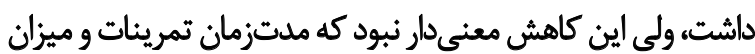

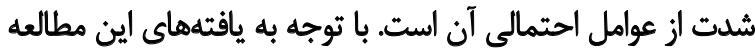

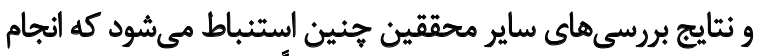

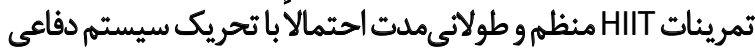

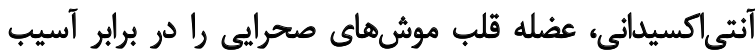

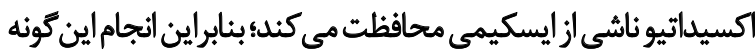

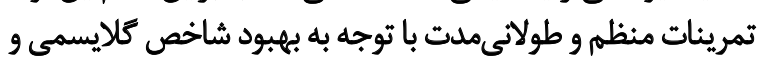

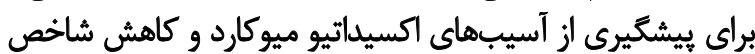

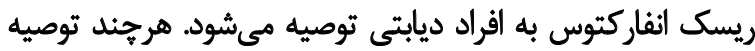
دقيقتر نيازمند انجام تحقيقات بيشتر در اين زمينه است دئ.

مالاحظات الخلاقي

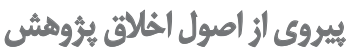

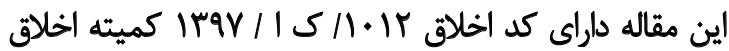

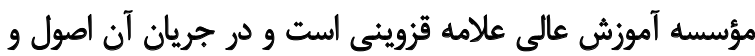

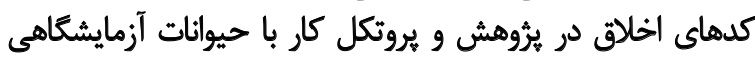

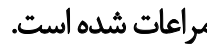

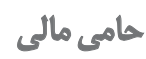

اين مقاله بركرفته از إياننامه كارشناسى ارشد خانم سيده 


\section{References}

[1] American Diabetes Association. Economic costs of diabetes in the U.S. in 2017. Diabetes Care. 2018; 41(5):917-28. [DOI:10.2337/ dci18-0007] [PMID] [PMCID]

[2] Rezende PC, Everett BM, Brooks MM, Vlachos H, Orchard TJ, Frye $\mathrm{RL}$, et al. Hypoglycemia and elevated troponin in patients with diabetes and coronary artery disease. J Am Coll Cardiol. 2018, 72(15):1778-86. [DOI:10.1016/j.jacc.2018.07.067] [PMID]

[3] American Diabetes Association. Classification and diagnosis of diabetes. Diabetes Care. 2016; 39(Suppl 1):S13-22. [DOI:10.2337/ dc16-S005]

[4] Liu Q, Wang Sh, Cai L. Diabetic cardiomyopathy and its mechanisms: Role of oxidative stress and damage. J Diabetes Investig. 2014; 5(6):623-34. [DOI:10.1111/jdi.12250] [PMID] [PMCID]

[5] Karar T, Elfaki EM, Qureshi Sh. Determination of the serum levels of troponin I and creatinine among Sudanese type 2 diabetes mellitus patients. J Nat Sci Biol Med. 2015; 6(Suppl 1):S80-4. [DOI:10.4103/0976-9668.166092] [PMID] [PMCID]

[6] Agewall S, Giannitsis E, Jernberg T, Katus H. Troponin elevation in coronary vs. non-coronary disease. Eur Heart J. 2011; 32(4):404 11. [DOI:10.1093/eurheartj/ehq456] [PMID]

[7] Bellia C, Lombardo M, Della-Morte D. Use of troponin as a predictor for cardiovascular diseases in patients with type 2 diabetes mellitus. Clin Chim Acta. 2020; 507:54-61. [DOI:10.1016/j. cca.2020.04.007] [PMID]

[8] Eggers KM, Venge P, Lindahl B, Lind L. Cardiac troponin I levels measured with a high-sensitive assay increase over time and are strong predictors of mortality in an elderly population. J Am Coll Cardiol. 2013; 61(18):1906-13. [DOI:10.1016/j.jacc.2012.12.048] [PMID]

[9] Apple FS, Collinson PO, IFCC Task Force on Clinical Applications of Cardiac Biomarkers. Analytical characteristics of high-sensitivity cardiac troponin assays. Lab Med Online. 2014; 4(1):55-62. [In Korean] [DOI:10.3343/Imo.2014.4.1.55]

[10] Jaffe AS. In search of specificity: The troponins. ACC Curr J Rev. 1995; 4(1):29-33. [DOI:10.1016/1062-1458(95)94006-L]

[11] Wells SM, Sleeper M. Cardiac troponins. J Vet Emerg Crit Care. 2008; 18(3):235-45. [DOI:10.1111/j.1476-4431.2008.00307.x]

[12] Shafer KM, Janssen L, Carrick-Ranson G, Rahmani S, Palmer D, Fujimoto $\mathrm{N}$, et al. Cardiovascular response to exercise training in the systemic right ventricle of adults with transposition of the great arteries. J Physiol. 2015; 593(11):2447-58. [DOI:10.1113/ JP270280] [PMID] [PMCID]

[13] Sasidharan SR, Joseph JA, Anandakumar S, Venkatesan V, Madhavan CNA, Agarwal A. An experimental approach for selecting appropriate rodent diets for research studies on metabolic disorders. Biomed Res Int. 2013; 2013:752870. [DOI:10.1155/2013/752870] [PMID] [PMCID]

[14] Asgari Hazaveh D, Riyahi Malayeri Sh, Babaei S. Effect of eight weeks high intensity interval training and medium intensity interval training and Aloe vera intake on serum vaspin and insulin resistance in diabetic male rats. J Arak Univ Med Sci. 2018; 20(11):6775. [In Persian] http://jams.arakmu.ac.ir/article-1-5406-en.htm
[15] Thomas C, Bishop D, Moore-Morris T, Mercier J. Effects of high-intensity training on MCT1, MCT4, and NBC expressions in rat skeletal muscles: Influence of chronic metabolic alkalosis. Am J Physiol Endocrinol Metab. 2007; 293(4):E916-22. [DOI:10.1152/ajpendo.00164.2007] [PMID]

[16] Long AN, Dagogo-Jack S. Comorbidities of diabetes and hypertension: Mechanisms and approach to target organ protection. J Clin Hypertens. 2011; 13(4):244-51. [DOI:10.1111/ j.1751-7176.2011.00434.x] [PMID] [PMCID]

[17] Umpierre D, Ribeiro PAB, Kramer CK, Leitão CB, Zucatti ATN, Azevedo MJ, et al. Physical activity advice only or structured exercise training and association with $\mathrm{HbA1c}$ levels in type 2 diabetes: A systematic review and meta-analysis. JAMA. 2011 305(17):1790-9. [DOI:10.1001/jama.2011.576] [PMID]

[18] Burgomaster KA, Howarth KR, Phillips SM, Rakobowchuk M, MacDonald MJ, McGee SL, et al. Similar metabolic adaptations during exercise after low volume sprint interval and traditional endurance training in humans. J Physiol. 2008; 586(1):151-60. [DOI:10.1113/jphysiol.2007.142109] [PMID] [PMCID]

[19] Alvarez C, Ramirez-Campillo R, Martinez-Salazar C, Mancilla $\mathrm{R}$, Flores-Opazo M, Cano-Montoya J, et al. Low-volume highintensity interval training as a therapy for type 2 diabetes. Int Sports Med. 2016; 37(9):723-9. [DOI:10.1055/s-0042-104935] [PMID]

[20] Francois ME, Little JP. Effectiveness and safety of high-intensity interval training in patients with type 2 diabetes. Diabetes Spectr. 2015; 28(1):39-44. [DOI:10.2337/diaspect.28.1.39] [PMID] [PMCID]

[21] Babuin L, Jaffe AS. Erratum: Troponin: The biomarker of choice for the detection of cardiac injury (Canadian Medical Association Journal (2005) 173, 10 (1191-1202)). CMAJ. 2006 174(3):353. https://mayoclinic.pure.elsevier.com/en/publications/erratum-troponin-the-biomarker-of-choice-for-thedetection-of-car-2

[22] Badole SL, Chaudhari SM, Jangam GB, Kandhare AD, Bodhankar SL. Cardioprotective activity of Pongamia pinnata in streptozotocin-nicotinamide induced diabetic rats. Biomed Res Int. 2015; 2015:403291. [DOI:10.1155/2015/403291] [PMID] [PMCID]

[23] Reasner CA. Reducing cardiovascular complications of type 2 diabetes by targeting multiple risk factors. J Cardiovasc Pharmacol. 2008; 52(2):136-44. [DOI:10.1097/FJC.0b013e31817ffe5a] [PMID]

[24] Zhou G, Li X, Hein DW, Xiang X, Marshall JP, Prabhu SD, et al. Metallothionein suppresses angiotensin II-induced nicotinamide adenine dinucleotide phosphate oxidase activation, nitrosative stress, apoptosis, and pathological remodeling in the diabetic heart. J Am Coll Cardiol. 2008; 52(8):655-66. [DOI:10.1016/j.jacc.2008.05.019] [PMID]

[25] Kumar SD, Vijaya M, Samy RP, Dheen ST, Ren M, Watt F, et al. Zinc supplementation prevents cardiomyocyte apoptosis and congenital heart defects in embryos of diabetic mice. Free Radic Biol Med. 2012; 53(8):1595-606. [DOI:10.1016/j. freeradbiomed.2012.07.008] [PMID]

[26] Marefati H, Aminizadeh S, Najafipour H, Dabiri Sh, Shahouzehi B. The effects of moderate-intensity interval training on the 
resistance to induced cardiac ischemia in adult male rats. Qom Univ Med Sci J. 2016; 10(4):1-9. [In Persian] http://journal. muq.ac.ir/article-1-923-en.html

[27] Löwbeer C, Seeberger A, Gustafsson SA, Bouvier F, Hulting J. Serum cardiac troponin T, troponin I, plasma BNP and left ventricular mass index in professional football players. J Sci Med Sport. 2007; 10(5):291-6. [DOI:10.1016/j.jsams.2006.10.002] [PMID]

[28] Sato Y, Kita T, Takatsu Y, Kimura T. Biochemical markers of myocyte injury in heart failure. Heart. 2004; 90(10):1110-3. [DOI:10.1136/hrt.2003.023895] [PMID] [PMCID]

[29] Gatta L, Armani A, lellamo F, Consoli C, Molinari F, Caminiti $G$, et al. Effects of a short-term exercise training on serum factors involved in ventricular remodelling in chronic heart failure patients. Int J Cardiol. 2012; 155(3):409-13. [DOI:10.1016/j. ijcard.2010.10.045] [PMID]

[30] Lee IM, Sesso HD, Paffenbarger Jr RS. Physical activity and coronary heart disease risk in men: Does the duration of exercise episodes predict risk? Circulation. 2000; 102(9):981-6. [DOI:10.1161/01.CIR.102.9.981] [PMID]

[31] French JP, Quindry JC, Falk DJ, Staib JL, Lee Y, Wang KKW, et al. Ischemia-reperfusion-induced calpain activation and SERCA2a degradation are attenuated by exercise training and calpain inhibition. Am J Physiol Heart Circ Physiol. 2006; 290(1):H12836. [DOI:10.1152/ajpheart.00739.2005] [PMID]

[32] Tofighi A, Ebrahimi Kalan A, Jamali Qarakhanlou B. The effect of resveratrol supplementation and aerobic training on cardiac tissue alteration of rats with acute myocardial infarction. Iran J Physiol Pharmacol. 2017; 1(4):211-21. [In Persian] http://ijpp. phypha.ir/article-1-273-en.html

[33] Powers SK, Smuder AJ, Kavazis AN, Quindry JC. Mechanisms of exercise-induced cardioprotection. Physiology. 2014; 29(1):2738. [DOI:10.1152/physiol.00030.2013] [PMID] [PMCID]

[34] Brouwers O, de Vos-Houben JMJ, Niessen PMG, Miyata T, van Nieuwenhoven F, Janssen BJA, et al. Mild oxidative damage in the diabetic rat heart is attenuated by glyoxalase-1 overexpression. Int J Mol Sci. 2013; 14(8):15724-39. [DOI:10.3390/ ijms140815724] [PMID] [PMCID] 
This Page Intentionally Left Blank 